\title{
On an SDP relaxation for kissing number
}

\author{
Jon Lee • Leo Liberti
}

Received: date / Accepted: date

\begin{abstract}
We demonstrate that an earlier semidefinite-programming relaxation for the kissing-number problem cannot provide good upper bounds. Furthermore, we show the existence of an optimal solution for this relaxation that cannot be used as a basis for establishing a good lower bound.
\end{abstract}

Mathematics Subject Classification (2010) 52C17

\section{Introduction}

The kissing number in dimension $d \geq 2$ is the maximum number $\mathrm{kn}(d)$ of euclidean unit balls arranged touching a central unit ball in such a way that the intersection of the interiors of any pair of balls in the configuration is empty. Formally, it is the maximum $n$ for which there exist $x_{1}, x_{2}, \ldots, x_{n} \in \mathbb{R}^{d}$ such that:

$$
\begin{aligned}
& \left\|x_{i}\right\|_{2}=1, \text { for } 1 \leq i \leq n \\
& \left\|x_{i}-x_{j}\right\|_{2} \geq 1, \text { for } 1 \leq i<j \leq n,
\end{aligned}
$$

where $x_{i}$ is the point of contact of the $i$-th surrounding ball with the central unit ball, for each $1 \leq i \leq n$.

J. Lee was supported in part by ONR grant N00014-17-1-2296. Additionally, part of this work was done while J. Lee was visiting the Simons Institute for the Theory of Computing. It was partially supported by the DIMACS/Simons Collaboration on Bridging Continuous and Discrete Optimization through NSF grant \#CCF-1740425.

\section{J. Lee}

Dept. of IOE, University of Michigan, Ann Arbor, Michigan, USA

E-mail: jonxlee@umich.edu

L. Liberti

CNRS LIX, École Polytechnique, 91128 Palaiseau, France

E-mail: liberti@lix.polytechnique.fr 
Exact values for $\mathrm{kn}(d)$ are known only for $d=2,3,4,8,24$; in particular, the determinations of $k n(3)=12$ (see [15]) and $k n(4)=24$ (see [12]) are fairly recent. Calculating other kissing numbers and sharpening lower and upper bounds is a subject of intense investigation (see [5, 14, 16, 16).

Lower bounds for kissing numbers (typically via finding good feasible configurations) have been computed using lattice theory (see [6]), as well as using mathematical-optimization formulations (see [10]) and global-optimization methods to solve them (see 8 ). The main tool for computing upper bounds (typically via solving relaxations) is Delsarte's linear program and its numerous variants, including mixed-combinatorial arguments (see [2,12]), generalizations (see [13]), and semidefinite-programming (SDP) extensions (see [3, 11).

Following [10,8, 9, we formulate the kissing-number problem as the following quadratically-constrained optimization problem:

$(\mathrm{KNP})$

$$
\begin{aligned}
v(n, d):= & \max \alpha \\
& \quad \text { subject to: } \\
& \left\|x_{i}\right\|_{2}^{2}=1, \text { for } 1 \leq i \leq n ; \\
& \left\|x_{i}-x_{j}\right\|_{2}^{2} \geq \alpha, \text { for } 1 \leq i<j \leq n ; \\
& -1 \leq x_{\ell} \leq 1, \text { for } 1 \leq \ell \leq d, 1 \leq i \leq n ; \\
& \alpha \geq 0,
\end{aligned}
$$

where $n, d \geq 2$ are given integers, $\alpha$ is a scalar decision variable, and $x:=$ $\left[x_{1}, x_{2}, \ldots, x_{n}\right]$ is a $d \times n$ matrix where the $i$-th column is $x_{i}$, representing the point of contact of the $i$-th surrounding ball with the central unit ball (for each $1 \leq i \leq n)$.

The variable $\alpha$ represents the minimum distance between pairs of (contact) points in a configuration. Solving this global-optimization problem and obtaining an optimal $\alpha \geq 1$ yields a proof that $\operatorname{kn}(d) \geq n$. Likewise, $\alpha<1$ implies that $\mathrm{kn}(d)<n$. So if we can solve instances of $[\mathrm{KNP})$, we can calculate kissing numbers.

Unfortunately current global-optimization software can only tackle (KNP for small values of $n$ and $d$ (see [8]) - certainly not for any values that can improve the current state of knowledge for bounding kissing numbers.

In what follows, we prove a theorem that provides an optimal solution of the natural SDP relaxation of (KNP in a closed form. In doing so, we will see that the associated upper bound is useless and the solution that we find cannot even be useful to construct good feasible configurations (i.e., as a lower bound).

\section{The useless SDP relaxation}

We consider the natural SDP relaxation of $(\mathrm{KNP}$ from $[9$ (also see [8]).

$$
v(n):=\max \alpha
$$


subject to:

$$
\begin{aligned}
& X_{i i}=1, \text { for } 1 \leq i \leq n ; \\
& -1 \leq X_{i j} \leq 1, \text { for } 1 \leq i \neq j \leq n ; \\
& X_{i i}+X_{j j}-X_{i j}-X_{j i} \geq \alpha, \text { for } 1 \leq i<j \leq n ; \\
& \alpha \geq 0 ; \\
& X \succeq 0 .
\end{aligned}
$$

We can see the relationship between $x$ in $(\mathrm{KNP})$ and $X$ in $(\mathrm{P})$, by defining $X:=x^{\prime} x$, which is then relaxed to the convex constraint $X-x^{\prime} x \succeq 0$. But because $x$ appears nowhere else in $(\mathrm{P})$, there is no further deterioration in the optimal objective value by instead simply enforcing the convex constraint $X \succeq 0$.

The fatal flaw for $(\mathrm{P})$ is that $d$ has disappeared, and so $x^{\prime} x$, which has rank no more than $d$, is replaced by $X$, which may have rank as high as $n$. Still, we have $v(n) \geq v(n, d)$, for all $d$, and so if $v(n)<1$, then the kissing number for all $d$ is less than $n$. But it is obvious that $\mathrm{kn}(d)$ grows with $d$, so this cannot happen. Hence, it is clear that $v(n) \geq 1$. This already shatters any hope of determining nontrivial upper bounds for kissing numbers using $(\mathrm{P})$. In fact, with Theorem 1, we demonstrate that $v(n)$ is precisely $2 n /(n-1)$.

On the other hand, we could hope that we might derive from an optimal solution $\tilde{X} \in \mathbb{R}^{n \times n}$ a good configuration $\tilde{x}=\left[\tilde{x}_{1}, \tilde{x}_{2}, \ldots, \tilde{x}_{n}\right] \in \mathbb{R}^{d \times n}$ using heuristic methods. Valid configurations provide lower bounds to kissing numbers. One possible idea is to employ principal component analysis (PCA) (see 7]) to derive an initial (probably infeasible) configuration and then a local nonlinear-optimization solver to seek a feasible configuration. We recall that PCA selects the $d$ coordinates corresponding to the largest eigenvalues of an $n \times n$ matrix. Even this further hope, for $(\mathrm{P})$ to be useful in the computation of lower bounds, is shattered by our proof of Theorem 1 1 which establishes that the associated optimal solution $\tilde{X}$ that we find for $(\mathrm{P})$ has rank $n-1$, and, fatally for the heuristic based on PCA, the multiplicity of the largest eigenvalue of $\tilde{X}$ is $n-1$.

Theorem 1 For all $n \geq 2, v(n)=2 n /(n-1)$.

Proof For the purpose of writing $(\mathrm{P})$ in a canonical form, we define some matrices in $\mathbb{R}^{n \times n}$. For $1 \leq i, j \leq n$, we let $E_{i j} \in \mathbb{R}^{n \times n}$ be all 0 except for a 1 in position $(i, j)$.

With these matrices in hand, we can recast $(\mathrm{P})$ in a type of canonical form as:

$\left(\mathrm{P}^{\prime}\right) \quad \max \alpha$

subject to:

dual var.

$$
\begin{aligned}
& \left\langle E_{i i}, X\right\rangle=1, \text { for } 1 \leq i \leq n ; \\
& \left\langle+E_{i j}+E_{j i}, X\right\rangle \leq 2, \text { for } 1 \leq i<j \leq n ; \\
& \left\langle-E_{i j}-E_{j i}, X\right\rangle \leq 2, \text { for } 1 \leq i<j \leq n ;
\end{aligned}
$$

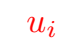

$y_{i j}$

$z_{i j}$ 
$\left\langle-E_{i i}-E_{j j}+E_{i j}+E_{j i}, X\right\rangle+\alpha \leq 0$, for $1 \leq i<j \leq n ; \quad w_{i j}$

$X \succeq 0, \alpha \geq 0$.

Using the dual variables indicated above, the dual of $\left(\mathrm{P}^{\prime}\right)$ is

$\left(\mathrm{D}^{\prime}\right) \min \sum_{i} u_{i}+2 \sum_{i<j}\left(y_{i j}+z_{i j}\right)$

subject to:

$$
\begin{aligned}
& \sum_{i=1}^{n} u_{i} E_{i i}+\sum_{i<j}\left[\left(y_{i j}-z_{i j}\right)\left(E_{i j}+E_{j i}\right)+w_{i j}\left(-E_{i i}-E_{j j}+E_{i j}+E_{j i}\right)\right] \succeq 0 ; \\
& \sum_{i<j} w_{i j} \geq 1 ; \\
& y_{i j}, z_{i, j}, w_{i, j} \geq 0, \text { for } 1 \leq i<j \leq n .
\end{aligned}
$$

It is compelling to simplify $\mathrm{D}^{\prime}$ to

(D)

$$
\min \sum_{i} u_{i}+2 \sum_{i<j}\left|\rho_{i j}\right|
$$

subject to:

$$
\begin{aligned}
& \sum_{i=1}^{n} u_{i} E_{i i}+\sum_{i<j}\left[\rho_{i j}\left(E_{i j}+E_{j i}\right)+w_{i j}\left(-E_{i i}-E_{j j}+E_{i j}+E_{j i}\right)\right] \succeq 0 \\
& \sum_{i<j} w_{i j} \geq 1
\end{aligned}
$$

(8) $w_{i, j} \geq 0$, for $1 \leq i<j \leq n$.

We will demonstrate that $v(n)=2 n /(n-1)$ by displaying feasible solutions to $(\mathrm{P})$ and $(\mathrm{D})$, both having objective value $2 n /(n-1)$.

Letting $J$ be an $n \times n$ matrix of all ones, our primal solution has $\alpha$ equal to $2 n /(n-1)$ and $X$ equal to $\frac{n}{n-1} I-\frac{1}{n-1} J$. We can see that we have $X_{i i}=$ $X_{j j}=1$ and $X_{i j}=X_{j i}=-\frac{1}{n-1}$, so clearly (1) is satisfied, (2) is satisfied (with slack everywhere), and we can readily check that (3) is satisfied as an equation (for all $i \neq j$ ). Finally, it is easy to see that this solution matrix $X$ is positive semidefinite, because the eigenvalues of $\frac{n}{n-1} I-\frac{1}{n-1} J$ are: $0, \frac{n}{n-1}, \ldots, \frac{n}{n-1}$.

Our dual solution has all $u_{i}$ equal to $2 /(n-1)$, all $w_{i j}$ equal to $1 /\left(\begin{array}{l}n \\ 2\end{array}\right)=$ $2 /(n(n-1))$, and all $\rho_{i j}$ equal to 0 . It is easy to see that the objective value of this dual solution is $\frac{2 n}{n-1}$, the same as the primal objective value.

Next, we need to check (6) and (7). For our particular dual solution, we can see (6) as

$$
\tilde{F}:=\sum_{i=1}^{n} u_{i} E_{i i}+\sum_{i<j} w_{i j}\left(-E_{i i}-E_{j j}+E_{i j}+E_{j i}\right) \succeq 0
$$


the left-hand side of which is

$$
\begin{aligned}
& \frac{2}{n-1} \sum_{i=1}^{n} E_{i i}+\frac{1}{\left(\begin{array}{c}
n \\
2
\end{array}\right)} \sum_{i<j}\left(-E_{i i}-E_{j j}+E_{i j}+E_{j i}\right) \\
& =\frac{2}{n-1} I+\frac{1}{\left(\begin{array}{c}
n \\
2
\end{array}\right)}[-(n-1) I+(J-I)] \\
& =\frac{1}{\left(\begin{array}{c}
n \\
2
\end{array}\right)} J
\end{aligned}
$$

which is clearly positive semidefinite. Finally, it is easy to see that (7) is satisfied (as an equation).

Incidentally, it is easy to verify complementarity of the primal and dual solutions in the proof. In particular,

$$
\tilde{F} \tilde{X}=\left(\frac{1}{\left(\begin{array}{c}
n \\
2
\end{array}\right)} J\right)\left(\frac{1}{n-1}(n I-J)\right)=0 .
$$

As an obvious consequence of Theorem 1, we see that $\lim _{n \rightarrow \infty} v(n)=2$, which was the behavior computationally observed in 9 .

As we have already observed, we always have $v(n) \geq 1$, but our theorem sharpens this and also demonstrates, due to the form of the optimal $\tilde{X}$ that we find, that PCA - which chooses the $d$ coordinates corresponding to the largest eigenvalues of $\tilde{X}$ - cannot be effective: our solution $\tilde{X}$ has one zero eigenvalue and the remaining $n-1$ (positive) ones are all identical; so the computed basis for the corresponding $(n-1)$-dimensional eigenspace is arbitrary. Therefore, a heuristic based on applying PCA to our optimal solution of $(\mathrm{P})$ cannot be effective.

\section{Possible future work}

We have not made any attempt to characterize all optimal solutions of $(\mathrm{P})$ or to try and demonstrate that the solution that we obtained is the unique optimal solution. There may be some interest in carrying out such an analysis, and there are tools to do it (see [1], for example). In any case, a consequence of our work is that the best hope for exploiting the relaxation $(\sqrt{\mathrm{P}})$ should strengthen it.

On the other side, we have exploited the extreme symmetry of $(\mathrm{P})$ to get a closed-form solution of it. It might be an interesting mathematical exercise (with no import on the kissing-number problem) to see how far this idea can be pushed. We could replace $(3)$ in $(\mathrm{P})$ with

$$
X_{i i}+X_{j j}-X_{i j}-X_{j i} \geq \alpha, \text { for } 1 \leq i<j \leq n:\{i, j\} \in \mathcal{E},
$$

where $\mathcal{E}$ is the edge set of a graph on vertex set $\{1,2, \ldots, n\}$. We have been working with complete graphs, but it seems possible to still get a closed form solution if the graph is say a Johnson graph or perhaps other classes of (or even all) distance-transitive graphs (see [4] for example). 


\section{References}

1. F. Alizadeh, J.-P.A. Haeberly, and M.L. Overton, Complementarity and nondegeneracy in semidefinite programming, Mathematical Programming 77 (1997), no. 1, 111-128.

2. K.M. Anstreicher, The thirteen spheres: a new proof, Discrete \& Computational Geometry 31 (2004), no. 4, 613-625.

3. C. Bachoc and F. Vallentin, New upper bounds for kissing numbers from semidefinite programming, Journal of the American Mathematical Society 21 (2008), 909-924.

4. N. Biggs, Algebraic graph theory, 2 ed., Cambridge Mathematical Library, Cambridge University Press, 1974

5. P. Boyvalenkov, S. Dodunekov, and O. Musin, A survey on the kissing numbers, Serdica Mathematical Journal 38 (2012), 507-522.

6. J. Conway and N. Sloane (eds.), Sphere packings, lattices and groups, Springer, Berlin, 1993.

7. I. Jolliffe, Principal component analysis, 2nd ed., Springer, Berlin, 2010.

8. S. Kucherenko, P. Belotti, L. Liberti, and N. Maculan, New formulations for the kissing number problem, Discrete Applied Mathematics 155 (2007), no. 14, 1837-1841.

9. L. Liberti, Mathematical programming bounds for kissing numbers, Optimization and Decision Science: Methodologies and Applications (A. Sforza and C. Sterle, eds.) Springer International Publishing, 2017, pp. 213-222.

10. N. Maculan, P. Michelon, and J. MacGregor Smith, Bounds on the kissing numbers in $\mathbb{R}^{n}$ : Mathematical programming formulations, Tech. report, University of Massachusetts, Amherst, USA, 1996.

11. H. Mittelmann and F. Vallentin, High-accuracy semidefinite programming bounds for kissing numbers, Experimental Mathematics 19 (2010), no. 2, 175-179.

12. O. Musin, The kissing number in four dimensions, Annals of Mathematics 168 (2008), $1-32$

13. F. Pfender, Improved Delsarte bounds for spherical codes in small dimensions, Journal of Combinatorial Theory, Series A 114 (2007), no. 6, 1133-1147.

14. F. Pfender and G. Ziegler, Kissing numbers, sphere packings and some unexpected proofs, Notices of the Americal Mathematical Society 51 (2004), no. 8, 873-883.

15. K. Schütte and B.L. van der Waerden, Das Problem der dreizehn Kugeln, Mathematische Annalen 125 (1953), 325-334.

16. G.G. Szpiro, Kepler's conjecture: How some of the greatest minds in history helped solve one of the oldest math problems in the world, John Wiley \& Sons, Inc., Hoboken, NJ, 2003. 